\title{
Sleep Quality and Electroencephalogram Delta Power
}

\author{
Siyu Long ${ }^{1,2}$, Rui Ding ${ }^{1,2}$, Junce Wang ${ }^{1,2}$, Yue Yu ${ }^{1}$, Jing Lu ${ }^{1,2 *}$ and Dezhong Yao ${ }^{1,2 *}$ \\ ${ }^{1}$ MOE Key Lab for Neuroinformation, The Clinical Hospital of Chengdu Brain Science Institute, University of Electronic \\ Science and Technology of China, Chengdu, China, ${ }^{2}$ School of Life Sciences and Technology, Center for Information \\ in Medicine, University of Electronic Science and Technology of China, Chengdu, China
}

\section{OPEN ACCESS}

Edited by:

Elena Urrestarazu,

University of Navarra Clinic, Spain

Reviewed by:

Agata Gabryelska,

Medical University of Lodz, Poland

${ }^{*}$ Correspondence:

Jing Lu

lujing@uestc.edu.cn

Dezhong Yao

dyao@uestc.edu.cn

Specialty section:

This article was submitted to

Sleep and Circadian Rhythms, a section of the journal

Frontiers in Neuroscience

Received: 28 October 2021 Accepted: 29 November 2021 Published: 15 December 2021

Citation:

Long S, Ding R, Wang J, Yu Y, Lu J and Yao D (2021) Sleep Quality and Electroencephalogram Delta

Power. Front. Neurosci. 15:803507.

doi: 10.3389/fnins.2021.803507
Delta activity on electroencephalogram (EEG) is considered a biomarker of homeostatic sleep drive. Delta power is often associated with sleep duration and intensity. Here, we reviewed the literature to explore how sleep quality was influenced by changes in delta power. However, we found that both the decrease and increase in delta power could indicate a higher sleep quality due to the various factors below. First, the differences in changes in delta power in patients whose sleep quality is lower than that of the healthy controls may be related to the different diseases they suffered from. We found that the patients mainly suffered from borderline personality disorder, and Rett syndrome may have a higher delta power than healthy individuals. Meanwhile, patients who are affected by Asperger syndrome, respiratory failure, chronic fatigue, and post-traumatic stress disorder have lower delta power. Second, if the insomnia patients received the therapy, the difference may be caused by the treatment method. Cognitive or music therapy shows that a better therapeutic effect is associated with decreased delta power, whereas in drug treatment, there is an opposite change in delta power. Last, for healthy people, the difference in delta change may be related to sleep stages. The higher sleep quality is associated with increased delta power during the NREM period, whereas a deceased delta change accompanies higher sleep quality during the REM period. Our work summarizes the effect of changes in delta power on sleep quality and may positively impact the monitoring and intervention of sleep quality.

Keywords: delta power, sleep quality, EEG, sleep stage, sleep disorder

Delta oscillation reflects the burst-pause firing pattern of the hyperpolarized thalamic cortex and corticothalamic neurons in synchronization (Steriade et al., 1993). Delta activity can be measured by delta power by Fourier analysis. It is a biomarker of homeostatic sleep drive, as evidence shows that delta power is enhanced after prolonged wakefulness, but declines as sleep deepens (Feinberg, 1974; Borbély et al., 1981; Achermann and Borbély, 2011). One study showed that after $40 \mathrm{~h}$ of sleep deprivation, both young and old subjects showed a significant increase in the power spectral density in the delta band (Münch et al., 2004). The finding that delta power is high during wake time but decreases during sleep (Esteban et al., 2005) was supported by healthy participants from various age groups as well as sleep-deprived people. A study of infants, adolescents, and adults showed that both children and adults experienced a gradual decrease in delta power and slow-wave sleep throughout the night (Bes et al., 1991). Sleep analysis of sleep-deprived young adults showed the gradual decline of electroencephalogram (EEG) power density in the low-frequency range as an indicator during sleep (Borbély et al., 1981). 
In addition, delta power is often accompanied by sleep duration and intensity (Davis et al., 2011). For example, after a lack of sleep, EEG delta power and non-rapid eye movement (NREM) duration during NREM episodes will increase (Pappenheimer et al., 1975; Achermann, 2004; Davis et al., 2011). Thus, delta power is a crucial part of the sleep process. We review some literature related to the change in delta power influencing sleep quality. However, current research on the relationship between delta power and sleep quality is inconclusive (Gao et al., 2020; Johnson and Durrant, 2021).

There is a lack of analysis on which factors lead to these opposite aspects. We list out the studies related to the change in delta power, including the advantages of decreased delta power and increased delta power (Tables 1, 2). We summarize the factors that may influence the adverse delta change. A wide range of factors, including the type, age, number, and sex of participants, the period of delta power changes, EEG derivation, and the power calculation method, may result in different conclusion.

We summarize the factors that may have influenced the contrary results. First, we infer that the category of the disease may influence the quality of sleep from various aspects, which may have different impacts on EEG power. We found that the patients in Table 1 mainly suffered from borderline personality disorder (BPD), Rett syndrome (RTT) (Lindberg et al., 2003; Philipsen et al., 2005; Ammanuel et al., 2015). A study of female patients with BPD who often have trouble sleeping showed that the delta power of BPD patients increased during NREM and REM sleep. Meanwhile, subjective scores, which mainly use the Pittsburgh Sleep Quality Index (PSQI) (Buysse et al., 1989), recorded a significant decline in sleep quality among BPD patients (Philipsen et al., 2005). It has also been suggested that sleep problems that present as reduced sleep duration, a longer incubation period, and interrupted sleep in girls (2-9 years old) with RTT were associated with higher delta frequency (Ammanuel et al., 2015). However, Table 2 mainly shows the patients who were affected by Asperger syndrome, respiratory failure, chronic fatigue, and post-traumatic stress disorder (PTSD) (Tani et al.'s, 2004; Guilleminault et al.'s, 2006; Kudchadkar et al., 2015; Wang et al., 2020). Tani et al.'s (2004) research mainly compared patients suffering from Asperger syndrome $(27.2 \pm 7.3)$ with healthy individuals and found a decreased delta power spectrum trend in patients during the SWS stage. The patients also had subjective impairment in the initiation and continuity of sleep. A study showed that children with respiratory failure who were associated with disruption of the normal sleep-wake rhythm had lower delta power than healthy children (Kudchadkar et al., 2015). Guilleminault et al.'s (2006) study compared the difference between chronic fatigue and normal and found that slow-wave sleep (SWS) percentage and sleep efficiency were lower, but there was a significant decrease in delta 2 relative power in the chronic fatigue group when compared to normal. Wang et al.'s (2020) study showed that compared with non-PTSD patients, PTSD patients exhibited reduced delta power during NREM sleep with reduced sleep depth. Second, the difference may be related to the method of treatment. Different therapies influence sleep quality in diverse ways. In studies of insomnia patients treated by cognitive behavioral therapy (CBT), the results showed that the continuous decrease in delta power was related to the treatment effect, and the rate of decrease in delta power was also used as the criterion of sleep drive (Feinberg, 1974; Krystal and Edinger, 2010). In addition, our previous study also found that SWS brain-wave music (Wu et al., 2009; Lu et al., 2012; Yao et al., 2020) leads to shorter sleep latency and higher sleep efficiency with decreased delta power (Gao et al., 2020). However, in Monti et al.'s (2000) study, power density in NREM sleep increased significantly in the low-frequency band $(0.25-1.0 \mathrm{~Hz})$ in the zolpidem group. We found that patients who received cognitive/music treatment and drug therapy had adverse delta changes (Monti et al.'s, 2000; Krystal and Edinger, 2010). Last, the period of delta change may influence the final results. We found from healthy participants that when the change in delta power occurred in the NREM period, better sleep quality was associated with increased delta power, which was proven in Kuula et al.'s (2020) study. They found that participants who received slow breathing training with higher sleep quality had higher delta power during N3, which is a deep sleep stage that lasts approximately $20-40 \mathrm{~min}$. There is also evidence showing that increases in the subjective stress burden were associated with decreases in delta power during NREM (Hall et al., 2000). Research in Park et al.'s (2021) found that exercise can lead to a short rapid eye movement latency and significantly increased delta power in SWS with enhanced SWS stability in early sleep phases. However, Gabryelska et al.'s (2019) study showed different situations in which better sleep quality was significantly associated with decreased Delta 1 power density in REM. Considering this fact, we speculate that the period of delta change may influence the impact of delta change.

Besides, we also listed some parameters about the delta power spectrum processing (Tables 1, 2) and wondered if these factors include the EEG duration per epoch, filter frequency, and the fast Fourier transform (FFT) window length might affect the final results. However, we did not find the common characteristic in each condition. Therefore, these parameters do not influence the relationship between delta power change and sleep quality.

The delta power is like a marker whose change can detect the sleep quality. The current research we have conducted mainly speculated the possible reason for the increase or the decrease of delta power indicates the better sleep quality. However, they are some speculations that we conclude by comparing the different studies. In the future, we should further explore the physiology mechanism behind these factors influencing the change of delta power and further influence sleep quality, which will provide more reliable evidence for the current study and draw a more precise conclusion on how the shift in delta power influences sleep quality.

In summary, we summarize the studies related to the change in delta power associated with sleep quality. We inferred that some factors might influence the difference in the impact of changes in delta power. We hope our research may contribute to a deeper understanding of the impact of delta power on monitoring and intervention of sleep quality. 
TABLE 1 | A delta power decrease indicates better sleep in different situations

\begin{tabular}{|c|c|c|c|c|c|c|c|c|c|c|c|}
\hline & $\begin{array}{l}\text { Participants } \\
\text { type }\end{array}$ & Sex & $\begin{array}{l}\text { Participants } \\
\text { amount }\end{array}$ & Age & Period $^{\mathrm{a}}$ & $\begin{array}{l}\text { EEG } \\
\text { derivations }\end{array}$ & $\begin{array}{l}\text { Calculation } \\
\text { index }\end{array}$ & $\begin{array}{l}\text { EEG } \\
\text { duration } \\
\text { per epoch }\end{array}$ & Filter & $\begin{array}{l}\text { FFT } \\
\text { window } \\
\text { length }\end{array}$ & Results \\
\hline $\begin{array}{l}\text { Philipsen et al., } \\
2005\end{array}$ & $\begin{array}{l}\text { borderline } \\
\text { personality } \\
\text { disorder } \\
\text { (BPD)/healthy }\end{array}$ & $\begin{array}{l}\text { Female/ } \\
\text { female }\end{array}$ & $20 / 20$ & $\begin{array}{c}28.6 \pm 7.88 / \\
\text { matched }\end{array}$ & All night & $\begin{array}{l}\text { C3-A2 and } \\
\text { C4-A1 }\end{array}$ & $\begin{array}{l}\text { power } \\
\text { spectrum }\end{array}$ & $30 \mathrm{~s}$ & $\begin{array}{l}70 \mathrm{~Hz} \\
\text { low-pass }\end{array}$ & $\begin{array}{l}512 \text { points } \\
(2.56 \mathrm{~s})\end{array}$ & $\begin{array}{l}\text { Patients with BDP had } \\
\text { lower NREM sleep and } \\
\text { showed increased delta } \\
\text { power throughout NREM } \\
\text { and REM. }\end{array}$ \\
\hline $\begin{array}{l}\text { Lindberg et al., } \\
2003\end{array}$ & $\begin{array}{l}\text { patients with } \\
\text { highly violent } \\
\text { offences }\end{array}$ & Male & 16 & $30.75 \pm 10.46$ & NREM & $\begin{array}{l}\mathrm{O} 2-\mathrm{P} 4 \text { and } \\
\mathrm{O} 1-\mathrm{P} 3\end{array}$ & $\begin{array}{l}\text { power } \\
\text { spectrum }\end{array}$ & $\begin{array}{l}\text { Not } \\
\text { mentioned }\end{array}$ & $\begin{array}{l}0.2-25 \mathrm{~Hz} \\
\text { band-pass }\end{array}$ & $\begin{array}{l}\text { Not } \\
\text { mentioned }\end{array}$ & $\begin{array}{l}\text { The patients who suffered } \\
\text { from BPD have higher delta } \\
\text { power and lower sleep } \\
\text { quality than the control } \\
\text { group. }\end{array}$ \\
\hline $\begin{array}{l}\text { Buysse et al., } \\
2008\end{array}$ & $\begin{array}{l}\text { primary } \\
\text { insomnia } \\
\text { (PI)/good } \\
\text { sleeper controls } \\
\text { (GSC) }\end{array}$ & $\begin{array}{l}\text { Mixedb/ } \\
\text { Mixed }^{b}\end{array}$ & $48 / 25$ & $\begin{array}{l}30.8 \pm 7.2 / \\
30.6 \pm 7.4\end{array}$ & NREM & $\begin{array}{l}\text { bilateral } \\
\text { central EEG } \\
\text { leads }\end{array}$ & $\begin{array}{l}\text { power } \\
\text { spectrum }\end{array}$ & $20 \mathrm{~s}$ & $\begin{array}{l}0.3-100 \mathrm{~Hz} \\
\text { band-pass } \\
(60 \mathrm{~Hz} \\
\text { notch) }\end{array}$ & $4 \mathrm{~s}$ & $\begin{array}{l}\text { The patients with primary } \\
\text { insomnia have higher delta } \\
\text { power compared with a } \\
\text { good sleeper. }\end{array}$ \\
\hline $\begin{array}{l}\text { Ammanuel } \\
\text { et al., } 2015\end{array}$ & $\begin{array}{l}\text { Rett syndrome } \\
(R T T) / \text { non-RTT }\end{array}$ & $\begin{array}{l}\text { Female/ } \\
\text { female }\end{array}$ & $10 / 15$ & $2-9$ & SWS & F3, C3, O1 & $\begin{array}{l}\text { power } \\
\text { spectrum }\end{array}$ & $10 \mathrm{~s}$ & $\begin{array}{l}1-70 \mathrm{~Hz} \\
\text { band-pass }\end{array}$ & $\begin{array}{l}\text { Not } \\
\text { mentioned }\end{array}$ & $\begin{array}{l}\text { The patients with RTT have } \\
\text { higher delta power, } \\
\text { accompanied by lower } \\
\text { sleep efficiency. }\end{array}$ \\
\hline $\begin{array}{l}\text { Münch et al., } \\
2004\end{array}$ & healthy & Mixed ${ }^{b}$ & $16 / 16$ & $\begin{array}{l}25 \pm 0.9 / \\
64.9 \pm 1.4\end{array}$ & $\begin{array}{l}\text { Not } \\
\text { mentioned }\end{array}$ & $\begin{array}{l}\mathrm{Fz}, \mathrm{Cz}, \mathrm{Pz} \\
\mathrm{Oz}\end{array}$ & $\begin{array}{l}\text { power } \\
\text { density }\end{array}$ & $20 \mathrm{~s}$ & $\begin{array}{l}30 \mathrm{~Hz} \\
\text { low-pass }\end{array}$ & $4 \mathrm{~s}$ & $\begin{array}{l}\text { After sleep deprivation, the } \\
\text { delta power density of the } \\
\text { youth and the old are both } \\
\text { increased. }\end{array}$ \\
\hline $\begin{array}{l}\text { Gabryelska } \\
\text { et al.'s, } 2019\end{array}$ & healthy & Mixed ${ }^{b}$ & 206 & $19-73$ & REM & $\begin{array}{l}\text { C3-A2 and } \\
\text { C4-A1 }\end{array}$ & $\begin{array}{l}\text { power } \\
\text { density }\end{array}$ & $30 \mathrm{~s}$ & $\begin{array}{l}\text { Not } \\
\text { mentioned }\end{array}$ & $\begin{array}{l}256 \text { points } \\
(2.5 \mathrm{~s}) \text { for } \\
102.4 \mathrm{~Hz} \\
\text { sample rate } \\
\text { and } 512 \\
\text { points } \\
(2.56 \mathrm{~s}) \text { for } \\
200 \mathrm{~Hz}\end{array}$ & $\begin{array}{l}\text { Better sleep quality is } \\
\text { significantly associated with } \\
\text { decreased Delta } 1 \text { power } \\
\text { density in REM. }\end{array}$ \\
\hline $\begin{array}{l}\text { Borbély et al., } \\
1981\end{array}$ & healthy & Mixed $^{b}$ & 8 & $21-29$ & $\begin{array}{l}\text { NREM- } \\
\text { REM sleep } \\
\text { cycles }\end{array}$ & $\begin{array}{l}\text { ipsilateral } \\
\text { fronto- } \\
\text { occipital } \\
\text { bipolar } \\
\text { derivation }\end{array}$ & $\begin{array}{l}\text { power } \\
\text { density }\end{array}$ & $30 \mathrm{~s}$ & $\begin{array}{l}25 \mathrm{~Hz} \\
\text { low-pass }\end{array}$ & $4 \mathrm{~s}$ & $\begin{array}{l}\text { During NREM-REM sleep } \\
\text { cycles, delta power density } \\
\text { declines. }\end{array}$ \\
\hline
\end{tabular}


TABLE 1 | (Continued)

\begin{tabular}{|c|c|c|c|c|c|c|c|c|c|c|c|}
\hline & $\begin{array}{l}\text { Participants } \\
\text { type }\end{array}$ & Sex & $\begin{array}{l}\text { Participants } \\
\text { amount }\end{array}$ & Age & Period $^{a}$ & $\begin{array}{l}\text { EEG } \\
\text { derivations }\end{array}$ & $\begin{array}{l}\text { Calculation } \\
\text { index }\end{array}$ & $\begin{array}{l}\text { EEG } \\
\text { duration } \\
\text { per epoch }\end{array}$ & Filter & $\begin{array}{l}\text { FFT } \\
\text { window } \\
\text { length }\end{array}$ & Results \\
\hline Dijk et al., 1990 & healthy & Male & 9 & $22-26$ & $\begin{array}{l}\text { NREM- } \\
\text { REM sleep } \\
\text { cycles }\end{array}$ & $\begin{array}{l}\text { C3-A2 and } \\
\text { C4-Al }\end{array}$ & $\begin{array}{l}\text { power } \\
\text { density }\end{array}$ & $20 s$ & $\begin{array}{l}25 \mathrm{~Hz} \\
\text { low-pass }\end{array}$ & $4 \mathrm{~s}$ & $\begin{array}{l}\text { Delta power spectrum } \\
\text { decreased during the first } \\
\text { three and four NREM-REM } \\
\text { cycles. }\end{array}$ \\
\hline $\begin{array}{l}\text { Ehlers and } \\
\text { Kupfer, } 1989\end{array}$ & healthy & Male & 24 & $21-70$ & NREM & C4 & $\begin{array}{l}\text { power } \\
\text { density }\end{array}$ & $60 s$ & $\begin{array}{l}0.3-30 \mathrm{~Hz} \\
\text { band-pass }\end{array}$ & $4 \mathrm{~s}$ & $\begin{array}{l}\text { The largest decrease in } \\
\text { delta activity occurred to } \\
\text { the greatest extent during } \\
\text { the first } 100 \text { min of sleep } \\
\text { (NREM period 1). }\end{array}$ \\
\hline $\begin{array}{l}\text { Krystal and } \\
\text { Edinger, } 2010\end{array}$ & $\begin{array}{l}\text { primary } \\
\text { insomnia } \\
\text { patients (PI) }\end{array}$ & Mixed $^{b}$ & 30 & $40-80$ & NREM & $\begin{array}{l}\mathrm{C} 3-\mathrm{M} 2 \text { and } \\
\mathrm{Oz}-\mathrm{Cz}\end{array}$ & $\begin{array}{l}\text { power } \\
\text { spectrum }\end{array}$ & $30 \mathrm{~s}$ & $\begin{array}{l}0.5-64 \mathrm{~Hz} \\
\text { band-pass }\end{array}$ & $2 \mathrm{~s}$ & $\begin{array}{l}\text { Patients treated with CBT } \\
\text { (cognitive behavior } \\
\text { treatment) has a faster } \\
\text { decrease in delta power } \\
\text { and a better } \\
\text { self-assessment of sleep } \\
\text { quality. }\end{array}$ \\
\hline
\end{tabular}

a Period, The period during which delta changes.

${ }^{b}$ Mixed-gender group of male and female. 
TABLE 2 | A delta power increase indicates better sleep in different situations.

\begin{tabular}{|c|c|c|c|c|c|c|c|c|c|c|c|}
\hline & $\begin{array}{l}\text { Participants } \\
\text { type }\end{array}$ & Sex & $\begin{array}{l}\text { Participants } \\
\text { amount }\end{array}$ & Age & Period $^{a}$ & $\begin{array}{l}\text { EEG } \\
\text { derivations }\end{array}$ & $\begin{array}{l}\text { Calculation } \\
\text { index }\end{array}$ & $\begin{array}{l}\text { EEG } \\
\text { duration } \\
\text { per epoch }\end{array}$ & Filter & $\begin{array}{l}\text { FFT } \\
\text { window } \\
\text { length }\end{array}$ & Results \\
\hline $\begin{array}{l}\text { Cohen et al., } \\
2018\end{array}$ & $\begin{array}{l}\text { preterm fetal } \\
\text { growth } \\
\text { restricted (FGR) } \\
\text { neonates/preterm } \\
\text { appropriate-for- } \\
\text { gestational-age } \\
\text { (AGA) } \\
\text { peers/healthy }\end{array}$ & $\begin{array}{l}\text { Not } \\
\text { mentioned }\end{array}$ & 18/20/19 & 1-6 mAVonths & $\begin{array}{l}\text { Not } \\
\text { mentioned }\end{array}$ & $\begin{array}{l}\mathrm{C} 3-\mathrm{M} 2 \text { and } \\
\mathrm{O} 1-\mathrm{M} 2 \text { or } \\
\mathrm{C} 4-\mathrm{M} 1 \text { and } \\
\mathrm{O} 2-\mathrm{M} 1\end{array}$ & $\begin{array}{l}\text { power } \\
\text { spectrum }\end{array}$ & $10 \mathrm{~s}$ & $\begin{array}{l}0.5-30 \mathrm{~Hz} \\
\text { band-pass }\end{array}$ & $4 \mathrm{~s}$ & $\begin{array}{l}\text { The delta power of } p \text {-FGR was } \\
\text { significantly reduced compared } \\
\text { to } p \text {-AGA infants. }\end{array}$ \\
\hline $\begin{array}{l}\text { Tani et al.'s, } \\
2004\end{array}$ & $\begin{array}{l}\text { Asperger } \\
\text { syndrome/healthy }\end{array}$ & $\begin{array}{l}\text { Not } \\
\text { mentioned }\end{array}$ & $20 / 10$ & $\begin{array}{l}27.2 \pm 7.3 / \\
26.5 \pm 8.1\end{array}$ & SWS & $\begin{array}{l}\text { C3-A1 and } \\
\text { C4-A1 }\end{array}$ & $\begin{array}{l}\text { power } \\
\text { spectrum }\end{array}$ & $30 \mathrm{~s}$ & $\begin{array}{l}0.5-45 \mathrm{~Hz} \\
\text { band-pass }\end{array}$ & $\begin{array}{l}512 \text { points } \\
(5.12 \mathrm{~s})\end{array}$ & $\begin{array}{l}\text { A statistically non-significant } \\
\text { trend toward decreased relative } \\
\text { delta power and increased } \\
\text { theta power in slow-wave sleep } \\
\text { was found in the AS group. }\end{array}$ \\
\hline $\begin{array}{l}\text { Kudchadkar } \\
\text { et al., } 2015\end{array}$ & $\begin{array}{l}\text { children with } \\
\text { respiratory } \\
\text { failure/healthy }\end{array}$ & $\begin{array}{l}\text { Not } \\
\text { mentioned }\end{array}$ & 8/unknown & $\begin{array}{c}\text { 6-16/ } \\
\text { unknown }\end{array}$ & $\begin{array}{l}\text { Not } \\
\text { mentioned }\end{array}$ & $\begin{array}{l}\text { bilateral } \\
\text { central and } \\
\text { occipital } \\
\text { EEG leads }\end{array}$ & $\begin{array}{l}\text { power } \\
\text { spectrum }\end{array}$ & $30 \mathrm{~s}$ & $\begin{array}{l}\text { Not } \\
\text { mentioned }\end{array}$ & $5 s$ & $\begin{array}{l}\text { Differences noted included } \\
\text { significantly lower mean } \\
\text { nighttime delta power in the } \\
\text { PICU patients compared to } \\
\text { healthy children. }\end{array}$ \\
\hline $\begin{array}{l}\text { Wang et al., } \\
2020\end{array}$ & $\begin{array}{l}\text { PTSD patient/ } \\
\text { non-PTSD }\end{array}$ & Mixed & $31 / 47$ & $\begin{array}{l}31.3 \pm 4.7 / \\
32.8 \pm 6.2\end{array}$ & NREM & $\begin{array}{l}\text { Not } \\
\text { mentioned }\end{array}$ & $\begin{array}{l}\text { power } \\
\text { spectrum }\end{array}$ & $5 s$ & $\begin{array}{l}0.5-50 \mathrm{~Hz} \\
\text { band-pass }\end{array}$ & $5 \mathrm{~s}$ & $\begin{array}{l}\text { Compared with non-PTSD, } \\
\text { PTSD exhibited reduced delta } \\
\text { power during NREM sleep. }\end{array}$ \\
\hline $\begin{array}{l}\text { Monti et al.'s, } \\
2000\end{array}$ & $\begin{array}{l}\text { insomniac } \\
\text { patients }\end{array}$ & Female & 12 & $27-59$ & $\begin{array}{l}\text { Not } \\
\text { mentioned }\end{array}$ & $\begin{array}{l}\text { Not } \\
\text { mentioned }\end{array}$ & $\begin{array}{l}\text { power } \\
\text { density }\end{array}$ & $20 \mathrm{~s}$ & $\begin{array}{l}0.25-30 \mathrm{~Hz} \\
\text { band-pass }\end{array}$ & $\begin{array}{l}\text { Not } \\
\text { mentioned }\end{array}$ & $\begin{array}{l}\text { All-night spectral analysis of the } \\
\text { EEG revealed that power } \\
\text { density in NREM sleep was } \\
\text { significantly increased in the } \\
\text { low-frequency band } \\
(0.25-1.0 \mathrm{~Hz} \text { ) in the zolpidem } \\
\text { group during the first 2-h } \\
\text { interval. }\end{array}$ \\
\hline Hall et al., 2000 & healthy & Mixed ${ }^{b}$ & 14 & $53 \pm 12$ & NREM & $\mathrm{C} 3$ or $\mathrm{C} 4$ & $\begin{array}{l}\text { power } \\
\text { density }\end{array}$ & $60 \mathrm{~s}$ & $\begin{array}{l}\text { Not } \\
\text { mentioned }\end{array}$ & $4 \mathrm{~s}$ & $\begin{array}{l}\text { Increases in subjective stress } \\
\text { burden were associated with } \\
\text { decreases in delta power. }\end{array}$ \\
\hline $\begin{array}{l}\text { Perrier et al., } \\
2015\end{array}$ & $\begin{array}{l}\text { primary } \\
\text { insomnia/ } \\
\text { healthy }\end{array}$ & $\begin{array}{l}\text { Mixed }^{b} / \\
\text { Mixed }^{b}\end{array}$ & $14 / 10$ & $\begin{array}{l}47 \pm 17 / \\
46 \pm 15\end{array}$ & NREM & $\begin{array}{l}\mathrm{FP} 1, \mathrm{FP} 2 \\
\mathrm{C} 3, \mathrm{C} 4 \\
\mathrm{O} 1, \mathrm{O} 2, \mathrm{~T} 3 \\
\mathrm{~T} 4\end{array}$ & $\begin{array}{l}\text { power } \\
\text { spectrum }\end{array}$ & $\begin{array}{l}\text { Not } \\
\text { mentioned }\end{array}$ & $\begin{array}{l}0.16-70 \mathrm{~Hz} \\
\text { band-pass }\end{array}$ & $5.12 \mathrm{~s}$ & $\begin{array}{l}\text { Primary insomnia patients } \\
\text { exhibited a lower delta power } \\
\text { spectrum compared to good } \\
\text { sleepers. }\end{array}$ \\
\hline $\begin{array}{l}\text { Guilleminault } \\
\text { et al.'s, } 2006\end{array}$ & $\begin{array}{l}\text { chronic fatigue/ } \\
\text { healthy }\end{array}$ & $\begin{array}{l}\text { Mixedb/ } \\
\text { Mixed }^{b}\end{array}$ & $14 / 14$ & $\begin{array}{l}41.1 \pm 9.8 / \\
33.6 \pm 10.2\end{array}$ & SWS & C4-A1 & $\begin{array}{l}\text { power } \\
\text { spectrum }\end{array}$ & $60 \mathrm{~s}$ & $\begin{array}{l}\text { Not } \\
\text { mentioned }\end{array}$ & $4 s$ & $\begin{array}{l}\text { Slow-wave sleep (SWS) } \\
\text { percentage and sleep efficiency } \\
\text { were lower, but there was a } \\
\text { significant decrease in delta } 2 \\
\text { relative power in the chronic } \\
\text { fatigue group when compared } \\
\text { to normals. }\end{array}$ \\
\hline
\end{tabular}


TABLE 2 | (Continued)

\begin{tabular}{|c|c|c|c|c|c|c|c|c|c|c|c|}
\hline & $\begin{array}{l}\text { Participants } \\
\text { type }\end{array}$ & Sex & $\begin{array}{l}\text { Participants } \\
\text { amount }\end{array}$ & Age & Perioda $^{a}$ & $\begin{array}{l}\text { EEG } \\
\text { derivations }\end{array}$ & $\begin{array}{l}\text { Calculation } \\
\text { index }\end{array}$ & $\begin{array}{l}\text { EEG } \\
\text { duration } \\
\text { per epoch }\end{array}$ & Filter & $\begin{array}{l}\text { FFT } \\
\text { window } \\
\text { length }\end{array}$ & Results \\
\hline $\begin{array}{l}\text { Kuula et al.'s, } \\
2020\end{array}$ & healthy & Mixed ${ }^{b}$ & 20 & $24.5 \pm 3.5$ & NREM & $\begin{array}{l}\mathrm{F} 3, \mathrm{~F} 4, \mathrm{C} 3, \\
\mathrm{C} 4, \mathrm{O} 3 \\
\mathrm{O} 4, \mathrm{~A} 1 \\
\text { and } \mathrm{A} 2\end{array}$ & $\begin{array}{l}\text { power } \\
\text { density }\end{array}$ & $30 \mathrm{~s}$ & $\begin{array}{l}0.5-35 \mathrm{~Hz} \\
\text { band-pass }\end{array}$ & $\begin{array}{l}1024 \text { points } \\
\text { (4 s) }\end{array}$ & $\begin{array}{l}\text { In the slow breathing condition } \\
\text { (sleep quality is higher), higher } \\
\text { central delta power during N3 } \\
\text { was observed. }\end{array}$ \\
\hline $\begin{array}{l}\text { Park et al.'s, } \\
2021\end{array}$ & healthy & Men & 9 & $23.8 \pm 0.7$ & SWS (N3) & $\begin{array}{l}\text { C3-A2, } \\
\text { C4-A1, } \\
\text { O2-A1, } \\
\text { O1-A2, } \\
\text { F3-A2, } \\
\text { F4-A1 }\end{array}$ & $\begin{array}{l}\text { power } \\
\text { spectrum }\end{array}$ & $30 \mathrm{~s}$ & $\begin{array}{l}\text { Not } \\
\text { mentioned }\end{array}$ & $5 \mathrm{~s}$ & $\begin{array}{l}\text { The group which experienced } \\
\text { exercise shows significantly } \\
\text { increased delta power in SWS } \\
\text { associated with increased SWS } \\
\text { stability and short REM latency. }\end{array}$ \\
\hline $\begin{array}{l}\text { Hoch et al., } \\
2001\end{array}$ & healthy & Mixed ${ }^{b}$ & 21 & $70-91$ & $\begin{array}{l}\text { Not } \\
\text { mentioned }\end{array}$ & $\begin{array}{l}\text { Not } \\
\text { mentioned }\end{array}$ & $\begin{array}{l}\text { Not } \\
\text { mentioned }\end{array}$ & $\begin{array}{l}\text { Not } \\
\text { mentioned }\end{array}$ & $\begin{array}{l}0.3-30 \mathrm{~Hz} \\
\text { band-pass }\end{array}$ & $\begin{array}{l}\text { Not } \\
\text { mentioned }\end{array}$ & $\begin{array}{l}\text { Participants in the } \\
\text { bed-restriction group showed a } \\
\text { median increase in sleep } \\
\text { efficiency of } 6.1 \text { versus } 1.8 \% \text { in } \\
\text { participants receiving sleep } \\
\text { hygiene instruction and an } \\
\text { increase in all-night delta EEG } \\
\text { power. }\end{array}$ \\
\hline $\begin{array}{l}\text { Manconi et al., } \\
2017\end{array}$ & $\begin{array}{l}\text { adults with a } \\
\text { diagnosis of } \\
\text { chronic primary } \\
\text { insomnia/ } \\
\text { healthy }\end{array}$ & $\begin{array}{l}\text { Not } \\
\text { mentioned }\end{array}$ & $20 / 13$ & Not mentioned & $\begin{array}{l}\text { NREM and } \\
\text { SWS }\end{array}$ & $\begin{array}{l}\text { C3-A2 or } \\
\text { C4-A1 }\end{array}$ & $\begin{array}{l}\text { power } \\
\text { spectrum }\end{array}$ & $2 \mathrm{~s}$ & $\begin{array}{l}0.1-35 \mathrm{~Hz} \\
\text { band-pass }\end{array}$ & $2 s$ & $\begin{array}{l}\text { During NREM sleep, patients } \\
\text { showed a clear decrease in the } \\
\text { relative power of the delta } \\
\text { band. }\end{array}$ \\
\hline $\begin{array}{l}\text { Izci-Balserak } \\
\text { et al., } 2018\end{array}$ & pregnancy & Female & 123 & $27.15 \pm 7.2$ & NREM & $\begin{array}{l}\text { C3-A2 and } \\
\text { C4-A1 }\end{array}$ & $\begin{array}{l}\text { power } \\
\text { spectrum }\end{array}$ & $30 \mathrm{~s}$ & $\begin{array}{l}0.3-100 \mathrm{~Hz} \\
\text { band-pass } \\
(60 \mathrm{~Hz} \\
\text { notch) }\end{array}$ & $4 \mathrm{~s}$ & $\begin{array}{l}\text { In late pregnancy, women had } \\
\text { shorter sleep duration, poorer } \\
\text { sleep efficiency, more } \\
\text { awakenings, more stage N2 } \\
\text { sleep, less slow-wave sleep, } \\
\text { less REM sleep, higher AHI, } \\
\text { and a higher periodic limb } \\
\text { movement index than early } \\
\text { pregnancy. Delta and theta } \\
\text { powers decreased. }\end{array}$ \\
\hline
\end{tabular}

a Period, The period during which delta changes.

${ }^{b}$ Mixed-gender group of male and female. 


\section{AUTHOR CONTRIBUTIONS}

SL, RD, JL, and DY conceived the study and drafted the manuscript. JW and YY collected the information. All authors made critical revisions to the work, which ultimately approved the upcoming edition, and agreed to be responsible for all aspects of the work.

\section{REFERENCES}

Achermann, P. (2004). The two-process model of sleep regulation revisited. Aviat. Space Environ. Med. 75(3 Suppl.), A37-A43.

Achermann, P., and Borbély, A. A. (2011). Sleep homeostasis and models of sleep regulation. Princip. Pract. Sleep Med. 2011, 431-444.

Ammanuel, S., Chan, W. C., Adler, D. A., Lakshamanan, B. M., Gupta, S. S., Ewen, J. B., et al. (2015). Heightened Delta Power during Slow-Wave-Sleep in Patients with Rett Syndrome Associated with Poor Sleep Efficiency. PLoS One 10:e0138113. doi: 10.1371/journal.pone.0138113

Bes, F., Schulz, H., Navelet, Y., and Salzarulo, P. (1991). The distribution of slowwave sleep across the night: a comparison for infants, children, and adults. Sleep 14, 5-12. doi: 10.1093/sleep/14.1.5

Borbély, A. A., Baumann, F., Brandeis, D., Strauch, I., and Lehmann, D. (1981). Sleep deprivation: effect on sleep stages and EEG power density in man. Electroencephalogr. Clin. Neurophysiol. 51, 483-495. doi: 10.1016/00134694(81)90225-x

Buysse, D. J., Germain, A., Hall, M. L., Moul, D. E., Nofzinger, E. A., Begley, A., et al. (2008). EEG spectral analysis in primary insomnia: NREM period effects and sex differences. Sleep 31, 1673-1682. doi: 10.1093/sleep/31.12. 1673

Buysse, D. J., Reynolds, C. F. III, Monk, T. H., Berman, S. R., and Kupfer, D. J. (1989). The Pittsburgh Sleep Quality Index: a new instrument for psychiatric practice and research. Psychiatry Res. 28, 193-213. doi: 10.1016/0165-1781(89) 90047-4

Cohen, E., Wong, F. Y., Wallace, E. M., Mockler, J. C., Odoi, A., Hollis, S., et al. (2018). EEG power spectrum maturation in preterm fetal growth restricted infants. Brain Res. 1678, 180-186. doi: 10.1016/j.brainres.2017.10. 010

Davis, C. J., Clinton, J. M., Jewett, K. A., Zielinski, M. R., and Krueger, J. M. (2011). Delta wave power: an independent sleep phenotype or epiphenomenon? J. Clin. Sleep Med. 7(5 Suppl.), S16-S18. doi: 10.5664/jcsm.1346

Dijk, D. J., Brunner, D. P., and Borbely, A. A. (1990). Time course of EEG power density during long sleep in humans. Am. J. Physiol. Regul. Integrat. Comparat. Physiol. 258, R650-R661. doi: 10.1152/ajpregu.1990.258.3.R650

Ehlers, C. L., and Kupfer, D. J. (1989). Effects of age on delta and REM sleep parameters. Electroencephalogr. Clin. Neurophysiol. 72, 118-125. doi: 10.1016/ 0013-4694(89)90172-7

Esteban, S., Nicolau, M. C., Gamundi, A., Akaarir, M., Rial, R. V., Parmeggiani, P. L., et al. (2005). Animal sleep: Phylogenetic correlations. Physiol. Nat. Sleep Lon. 2005, 207-245.

Feinberg, I. (1974). Changes in sleep cycle patterns with age. J. Psychiatr. Res. 10, 283-306. doi: 10.1016/0022-3956(74)90011-9

Gabryelska, A., Feige, B., Riemann, D., Spiegelhalder, K., Johann, A., Białasiewicz, P., et al. (2019). Can spectral power predict subjective sleep quality in healthy individuals? J. Sleep Res. 28:e12848. doi: 10.1111/jsr.12848

Gao, D., Long, S., Yang, H., Cheng, Y., Guo, S., Yu, Y., et al. (2020). SWS BrainWave Music May Improve the Quality of Sleep: An EEG Study. Front. Neurosci. 14:67. doi: 10.3389/fnins.2020.00067

Guilleminault, C., Poyares, D., Rosa, A., Kirisoglu, C., Almeida, T., and Lopes, M. C. (2006). Chronic fatigue, unrefreshing sleep and nocturnal polysomnography. Sleep Med. 7, 513-520. doi: 10.1016/j.sleep.2006.03.016

Hall, M., Buysse, D. J., Nowell, P. D., Nofzinger, E. A., Houck, P., Reynolds, C. F. III, et al. (2000). Symptoms of stress and depression as correlates of sleep in primary insomnia. Psychosom. Med. 62, 227-230. doi: 10.1097/00006842-20000300000014

Hoch, C. C., Reynolds, C. F. III, Buysse, D. J., Monk, T. H., Nowell, P., Begley, A. E., et al. (2001). Protecting sleep quality in later life: a pilot study of bed

\section{FUNDING}

This work was supported by the Project of Science and Technology Department of Sichuan Province (Nos. 2021YFS0135 and 2020ZYD013) and the Research Unit of NeuroInformation, Chinese Academy of Medical Sciences (No. 2019RU035).

restriction and sleep hygiene. J. Gerontol. B Psychol. Sci. Soc. Sci. 56, 52-59. doi: 10.1093/geronb/56.1.p52

Izci-Balserak, B., Keenan, B. T., Corbitt, C., Staley, B., Perlis, M., and Pien, G. W. (2018). Changes in Sleep Characteristics and Breathing Parameters During Sleep in Early and Late Pregnancy. J. Clin. Sleep Med. 14, 1161-1168. doi: $10.5664 / \mathrm{jcsm} .7216$

Johnson, J. M., and Durrant, S. J. (2021). Commentary: SWS Brain-Wave Music May Improve the Quality of Sleep: An EEG Study. Front. Neurosci. 15:609169. doi: $10.3389 /$ fnins.2021.609169

Krystal, A. D., and Edinger, J. D. (2010). Sleep EEG predictors and correlates of the response to cognitive behavioral therapy for insomnia. Sleep 33, 669-677. doi: $10.1093 /$ sleep/33.5.669

Kudchadkar, S. R., Yaster, M., Punjabi, A. N., Quan, S. F., Goodwin, J. L., Easley, R. B., et al. (2015). Temporal Characteristics of the Sleep EEG Power Spectrum in Critically Ill Children. J. Clin. Sleep Med. 11, 1449-1454. doi: 10.5664/jcsm. 5286

Kuula, L., Halonen, R., Kajanto, K., Lipsanen, J., Makkonen, T., Peltonen, M., et al. (2020). The Effects of Presleep Slow Breathing and Music Listening on Polysomnographic Sleep Measures - a pilot trial. Sci. Rep. 10:7427. doi: 10.1038/ s41598-020-64218-7

Lindberg, N., Tani, P., Appelberg, B., Naukkarinen, H., Rimón, R., PorkkaHeiskanen, T., et al. (2003). Human impulsive aggression: a sleep research perspective. J. Psychiatr. Res. 37, 313-324. doi: 10.1016/s0022-3956(03)00041-4

Lu, J., Wu, D., Yang, H., Luo, C., Li, C., and Yao, D. (2012). Scale-free brain-wave music from simultaneously EEG and fMRI recordings. PLoS One 7:e49773. doi: 10.1371/journal.pone.0049773

Manconi, M., Ferri, R., Miano, S., Maestri, M., Bottasini, V., Zucconi, M., et al. (2017). Sleep architecture in insomniacs with severe benzodiazepine abuse. Clin. Neurophysiol. 128, 875-881. doi: 10.1016/j.clinph.2017.03.009

Monti, J. M., Alvariño, F., and Monti, D. (2000). Conventional and power spectrum analysis of the effects of zolpidem on sleep EEG in patients with chronic primary insomnia. Sleep 23, 1075-1084.

Münch, M., Knoblauch, V., Blatter, K., Schröder, C., Schnitzler, C., Kräuchi, K., et al. (2004). The frontal predominance in human EEG delta activity after sleep loss decreases with age. Eur. J. Neurosci. 20, 1402-1410. doi: 10.1111/j.14609568.2004.03580.x

Pappenheimer, J. R., Koski, G., Fencl, V., Karnovsky, M. L., and Krueger, J. (1975). Extraction of sleep-promoting factor $\mathrm{S}$ from cerebrospinal fluid and from brains of sleep-deprived animals. J. Neurophysiol. 38, 1299-1311. doi: 10.1152/jn.1975. 38.6.1299

Park, I., Díaz, J., Matsumoto, S., Iwayama, K., Nabekura, Y., Ogata, H., et al. (2021). Exercise improves the quality of slow-wave sleep by increasing slow-wave stability. Sci. Rep. 11:4410. doi: 10.1038/s41598-021-83817-6

Perrier, J., Clochon, P., Bertran, F., Couque, C., Bulla, J., Denise, P., et al. (2015). Specific EEG sleep pattern in the prefrontal cortex in primary insomnia. PLoS One 10:e0116864. doi: 10.1371/journal.pone.0116864

Philipsen, A., Feige, B., Al-Shajlawi, A., Schmahl, C., Bohus, M., Richter, H., et al. (2005). Increased delta power and discrepancies in objective and subjective sleep measurements in borderline personality disorder. J. Psychiatr. Res. 39, 489-498. doi: 10.1016/j.jpsychires.2005.01.002

Steriade, M., McCormick, D. A., and Sejnowski, T. J. (1993). Thalamocortical oscillations in the sleeping and aroused brain. Science 262, 679-685. doi: 10. $1126 /$ science. 8235588

Tani, P., Lindberg, N., Nieminen-von Wendt, T., von Wendt, L., Virkkala, J., Appelberg, B., et al. (2004). Sleep in young adults with Asperger syndrome. Neuropsychobiology 50, 147-152. doi: 10.1159/000079106

Wang, C., Ramakrishnan, S., Laxminarayan, S., Dovzhenok, A., Cashmere, J. D., Germain, A., et al. (2020). An attempt to identify reproducible high-density 
EEG markers of PTSD during sleep. Sleep 43:zsz207. doi: 10.1093/sleep/zsz 207

Wu, D., Li, C. Y., and Yao, D. Z. (2009). Scale-free music of the brain. PLoS One 4:e5915. doi: 10.1371/journal.pone.0005915

Yao, D., Zhang, Y., Liu, T., Xu, P., Gong, D., Lu, J., et al. (2020). Bacomics: a comprehensive cross area originating in the studies of various brain-apparatus conversations. Cogn. Neurodyn. 14, 425-442. doi: 10.1007/s11571-020-09 $577-7$

Conflict of Interest: The authors declare that the research was conducted in the absence of any commercial or financial relationships that could be construed as a potential conflict of interest.
Publisher's Note: All claims expressed in this article are solely those of the authors and do not necessarily represent those of their affiliated organizations, or those of the publisher, the editors and the reviewers. Any product that may be evaluated in this article, or claim that may be made by its manufacturer, is not guaranteed or endorsed by the publisher.

Copyright (c) 2021 Long, Ding, Wang, Yu, Lu and Yao. This is an open-access article distributed under the terms of the Creative Commons Attribution License (CC BY). The use, distribution or reproduction in other forums is permitted, provided the original author(s) and the copyright owner(s) are credited and that the original publication in this journal is cited, in accordance with accepted academic practice. No use, distribution or reproduction is permitted which does not comply with these terms. 